\title{
Snail1 is positively correlated with atrial fibrosis in patients with atrial fibrillation and rheumatic heart disease
}

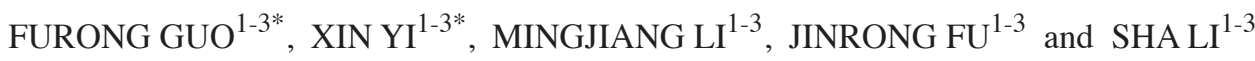 \\ ${ }^{1}$ Department of Cardiology, Renmin Hospital of Wuhan University; ${ }^{2}$ Cardiovascular Research Institute; \\ ${ }^{3}$ Hubei Key Laboratory of Cardiology, Wuhan University, Wuhan, Hubei 430060, P.R. China
}

Received September 23, 2016; Accepted June 5, 2017

DOI: $10.3892 / e t m .2017 .5084$

\begin{abstract}
The present study investigated the association between Snaill and atrial fibrosis in patients with atrial fibrillation (AF) and rheumatic heart disease (RHD) and to determine the possible mechanism underlying this interrelation. A total of 19 patients were included in the current study and were divided into two groups: A sinus rhythm (SR) group $(\mathrm{n}=9)$ and an AF group $(\mathrm{n}=10)$. All patients underwent heart valve replacement surgery, during which $\sim 200 \mathrm{mg}$ right atrium tissue was obtained. Hematoxylin and eosin and Masson's trichrome-stained sections were used to evaluate the morphological changes of cardiomyocytes and the level of fibrosis. Immunohistochemistry was applied to observe the location and expression of Snaill. Reverse transcription-quantitative polymerase chain reaction was used to measure Snaill mRNA levels. Western blotting was used to determine changes in the expression of Snaill, as well as in the expression of proteins involved in the Wnt pathway, including Wnt1, Wnt 3a, Wnt8a, Wnt5a and Wnt11. Compared with the SR group, expanded cardiomyocytes and higher collagen deposition was detected in the atrial tissue of the AF group. The expression of Snaill mRNA and protein was significantly higher in the AF group than in the SR group $(\mathrm{P}<0.05)$. Additionally, the expression of Wnt1, 3a and $8 \mathrm{a}$ in the canonical Wnt signaling pathway, and Wnt5a and 11 in the noncanonical Wnt signaling pathway were significantly increased in the AF group. Furthermore, the phosphorylation level of glycogen synthase kinase $3 \beta$ (GSK $3 \beta$ ) and the levels of $\beta$-catenin and GSK3 $\beta$ were significantly increased in the AF group compared with the SR group $(\mathrm{P}<0.05)$. Snail1 may be involved in the development and maintenance of atrial fibrosis in patients with atrial fibrillation and rheumatic heart
\end{abstract}

Correspondence to: Professor Mingjiang Li, Department of Cardiology, Renmin Hospital of Wuhan University, 238 Jiefang Road, Wuhan, Hubei 430060, P.R. China

E-mail:754851539@qq.com

${ }^{*}$ Contributed equally

Key words: Snaill, atrial fibrosis, Wnt signaling pathway, atrial fibrillation, rheumatic heart disease disease and may be developed as a novel biomarker to evaluate myocardial fibrosis in the future. Additionally, the current study suggests that the Wnt signaling pathway may participate in the process of increased Snaill expression and atrial fibrosis in patients with $\mathrm{AF}$ and $\mathrm{RHD}$.

\section{Introduction}

Atrial fibrillation (AF) is becoming more common in patients with cardiovascular diseases, including rheumatic heart disease (RHD), hypertension, coronary heart disease, congenital heart disease, cardiomyopathy and pericardial disease (1). Atrial fibrillation can severely affect human health; rapid ventricular rates may result in the development of fatal malignant arrhythmia, such as ventricular fibrillation (1). Another condition induced by $\mathrm{AF}$ is mural thrombus, which may lead to the development of major adverse cardiovascular events, including acute myocardial infarction and stroke (2). Previous studies have demonstrated that atrial structural remodeling occurs during the onset and development of atrial fibrillation, which leads to a dilated left atrium and a decline in left ventricular ejection fraction (3-7). However, preventing or improving myocardial fibrosis may significantly reduce the left atrial diameter (LAD) and improve cardiac function in patients with AF $(4,5,8)$. Therefore, blocking atrial fibrosis may be a better way of preventing the development and deterioration of atrial fibrillation.

Atrial fibrosis is mediated by cardiac fibroblasts, which can be transferred from local static cardiac fibroblasts, epithelial cells and bone marrow stem cells under pathological conditions, such as ischemic injury, anoxic injury and the stimulation of TGF- $\beta(2,9)$. Previous studies have demonstrated that epithelial and endothelial cells can be transformed into fibroblasts in a process known as the epithelial mesenchymal transition (EMT), which serves important roles in pulmonary fibrosis, renal fibrosis, cardiac hypertrophy and cardiac fibrosis (10-14). Snaill is an important regulator and specific marker of the EMT and contributes to the formation of many tissues during embryonic development and to the acquisition of invasive properties during epithelial carcinoma (15). It was determined that Snaill may be activated in the myocardium following myocardial infarction and that it moves from the endothelium to the mesenchymal cells of the coronary artery, reflecting the progression of the EMT (16). Therefore, it is hypothesized that the EMT may be one of the mechanisms involved in the 
occurrence and development of atrial fibrosis, and inhibiting the activity of Snaill may attenuate the EMT in patients with atrial fibrillation and therefore improve the symptoms of atrial fibrosis. Wnt signaling serves an important role in organ fibrosis, which is also involved in renal fibrosis, lung fibrosis and liver fibrosis (17-19). However, it remains unclear whether Wnt signaling participates in the atrial fibrosis activated by EMT that occurs in AF. Therefore, the current study aimed to investigate the association between Snaill and atrial fibrosis in patients with AF and RHD.

\section{Materials and methods}

Patients. Patients with RHD aged 30-60 years old planning to receive heart valve replacement surgery who were admitted to the Department of Cardiovascular Surgery, Renmin Hospital of Wuhan University (Wuhan, China) between June 2015 and April 2016 were recruited in the current study. Cardiac function was ranked between Class I and Class III using the New York Heart Association Functional Classification (1). Patients with coronary atherosclerotic heart disease, chronic pulmonary heart disease, infective endocarditis, hyperthyroidism, serious liver, kidney or lung dysfunction, or malignant tumor were excluded from the current study. Patients taking drugs that could attenuate ventricular remodeling, including angiotensin converting enzyme inhibitors, angiotensin-receptor blockers, $\beta$-receptor blockers and spironolactone were also excluded. Following an initial evaluation, 19 patients (10 males and 9 females) were enrolled in the current study. These patients were divided into two groups: An AF group (n=10) and a sinus rhythm (SR) group $(n=9)$. The general clinical characteristics of the patients are presented in Table I. The present study was approved by the Ethics Committee of Renmin Hospital of Wuhan University (Wuhan, China) and samples were obtained following the regulations of Renmin Hospital of Wuhan University. All patients provided written informed consent prior to their inclusion in the current study.

Reagents. Primary antibodies against Snail1 (cat. no. 125918) were purchased from GeneTex, Inc. (Irvine, CA, USA). Primary antibodies against Wnt1 (ab85060), Wnt8a (ab130930), phosphorylated glycogen synthase kinase $3 \beta$ (P-GSK3 $\beta$; ab130937) and GAPDH (ab37168) were obtained from Abcam (Cambridge,UK). Primary antibodies against Wnt3a (bs1700R) and Wnt5a (bs1948R) were purchased from BIOSS (Beijing, China). The primary antibody against Wnt11 (sc50360) was purchased from Santa Cruz Biotechnology, Inc. (Dallas, TX, USA). Antibodies against $\beta$-catenin (BA0426) and $\beta$-actin (BM0627) were obtained from Wuhan Boster Biological Technology, Ltd. (Wuhan, China). The antibody against GSK3 $\beta$ (22104-1-AP) was purchased from Wuhan Sanying Biotechnology (Wuhan, China). The bicinchoninic acid (BCA) protein assay kit was purchased from Beyotime Institute of Biotechnology (Haimen, China). TRIzol (252250AX) was obtained from Aidlab Biotechnologies Co., Ltd. (Beijing, China). M-MLV Reverse Transcriptase (RNase H; CO2010A) and $\mathrm{ddH}_{2} \mathrm{O}$ (DNase/RNase Free; C1D230A) were purchased from GeneCopoeia, Inc. (Rockville, MD, USA) and RNase Inhibitor (I21222) was obtained from TransGen Biotech, Inc. (Beijing, China).
Human myocardium sample collection. All patients underwent heart valve replacement surgery, during which time $200 \mathrm{mg}$ right atrium tissue was collected from each patient. Each sample was divided into two sections. One section was rapidly placed into a liquid nitrogen container and then placed in a refrigerator at $-80^{\circ} \mathrm{C}$; the other section was washed with saline solution and subsequently soaked in $4 \%$ paraformaldehyde solution under the room temperature for pathological analysis.

Hematoxylin and eosin $(H \& E)$ staining. H\&E staining was used to detect morphological changes in atrial myocytes. Right atrium tissues were fixed in $4 \%$ paraformaldehyde solution at room temperature for at least $24 \mathrm{~h}$ and embedded in paraffin wax. Sections $4-\mu \mathrm{m}$ thick were placed on clean, positively-charged microscope slides. Following drying, the slides were heated at $60^{\circ} \mathrm{C}$. Following deparaffinization and rehydration, the slides were washed, stained with hematoxylin for $7 \mathrm{~min}$, differentiated with $0.3 \%$ acid alcohol, rinsed in Scott's tap water substitute, stained with eosin for 2 min and then dehydrated, cleared and mounted. All the above experiments were performed at room temperature. A light microscope was used to visualize slides.

Masson's staining. Masson's staining was conducted to determine the collagen deposition of interstitial and perivascular in atrial myocardium. Following deparaffinization and rehydration, the slides were washed and re-fixed in Bouin's solution for $1 \mathrm{~h}$ at $56^{\circ} \mathrm{C}$ to improve staining quality. Slides were then stained with Weigert's iron hematoxylin for $5 \mathrm{~min}$, stained in Biebrich scarlet-acid fuchsin solution for $8 \mathrm{~min}$ and then stained in phosphomolybdic acid solution for $5 \mathrm{~min}$. Slides were subsequently stained with aniline blue solution and differentiated in $1 \%$ acetic acid, rapidly dehydrated, cleared in xylene and mounted using resinous mounting medium. All steps of Masson's staining were conducted at room temperature. A blue stain indicated the presence of collagen and a red stain indicated the presence of muscle and cytoplasm. High-magnification light micrographs were captured using light microscopy. Image-Pro Plus 6.0 (Media Cybernetics, Inc. Rockville, MD, USA) was used to determine the collagen volume fraction $(\mathrm{CVF})$.

Immunohistochemistry. Immunohistochemistry was used to measure the deposition of Snaill in myocardium tissues. Following deparaffinization and rehydration, EnVision ${ }^{\mathrm{TM}}$ two-step protocol, the tissue specimens were fixed in $4 \%$ formaldehyde solution at room temperature for at least $24 \mathrm{~h}$, embedded in paraffin and sliced at $4 \mu \mathrm{m}$ thickness. The paraffin was then removed. Bovine serum albumin (BSA) solution (3\%; Beijing Solarbio Science and Technology Co., Ltd., Beijing, China) was added to each slide until tissues were covered with BSA solution, and incubated for $30 \mathrm{~min}$ at room temperature. Subsequently, the slides were washed to get rid of the blocking solution and the primary antibody against Snaill (dilution, 1:50) was added for $50 \mathrm{~min}$ at room temperature. Following washing with phosphate-buffered saline, horseradish peroxidase-conjugated AffiniPure goat anti-rabbit antibody (cat. no. K5007; Dako; Agilent Technologies, Inc., Santa Clara, CA, USA) was added to the slides for $50 \mathrm{~min}$ at room temperature. 3,3'-diaminobenzidine was subsequently added 
as color reagent until the positive expression of brown-yellow appeared under the microscope (maximum of $10 \mathrm{~min}$ ). Then hematoxylin was used as a counterstain for $3 \mathrm{~min}$. Slides were then fixed with a neutral gum mount. All experiments in this part were performed at room temperature. A light microscope was used to observe the slides. Brown-yellow expression in myocardium tissue indicated positive expression of Snail1.

Reverse transcription-quantitative polymerase chain reaction $(R T-q P C R)$. The expression of Snaill was measured using RT-qPCR. Total RNA was isolated from the right atrium tissue using TRIzol reagent. A First Chain Synthesis kit for cDNA (Fermentas; Thermo Fisher Scientific, Inc., Waltham, MA, USA) was used for reverse transcription, according to the manufacturer's protocol. SYBR-Green/Fluorescein qPCR Master mix (2X) (Fermentas; Thermo Fisher Scientific, Inc.) and Ex Taq ${ }^{\mathrm{TM}}$ (Takara Bio, Inc., Otsu, Japan) were used for PCR. qPCR was performed on an Illumina-Eco machine (Illumina, Inc., San Diego, CA, USA). The result was normalized against $\beta$-actin gene expression. The sequences of all primers used in the current study were as follows: Snail1, forward, 5'-GCACAT CCGAAGCCACA-3' and reverse, 5'-GAGAAGGTCCGAGC ACA-3'; $\beta$-actin, forward, 5'-AGCGAGCATCCCCCAAAG TT-3' and reverse, 5'-GGGCACGAAGGCTCATCATT-3'. The reaction conditions were as follows: $50^{\circ} \mathrm{C}$ for $2 \mathrm{~min}, 95^{\circ} \mathrm{C}$ for $10 \mathrm{~min}, 95^{\circ} \mathrm{C}$ for $30 \mathrm{sec}, 60^{\circ} \mathrm{C}$ for $30 \mathrm{sec}$. RT-PCR experiments were performed with $1 \mathrm{~g}$ of total RNA, followed by 40 cycles of PCR amplification. The expression levels were quantified using changes in the fluorescence signal through the analysis of the $\mathrm{Cq}$ value and standard curve (20); the starting template was quantitatively analyzed with RUO ViiA ${ }^{\mathrm{TM}} 7$ software (Thermo Fisher Scientific, Inc.).

Western blot analysis. Total protein was extracted from the right atrium tissue using radioimmunoprecipitation assay lysis buffer (cat. no. P0013B; Beyotime Institute of Biotechnology). The BCA Protein assay kit was used to determine protein concentration. Denatured protein was loaded and separated using 10\% SDS-PAGE and then transferred to a nitrocellulose membrane. Following blocking with 5\% non-fat milk for $60 \mathrm{~min}$ at room temperature, the membrane was incubated with primary antibodies overnight at $4^{\circ} \mathrm{C}$. Primary antibodies were as follows: Snail1 (dilution, 1:3,000), Wnt1 (dilution, 1:1,000), Wnt3a (dilution, 1:500), Wnt5a (dilution, 1:500), Wnt8a (dilution, 1:1,000), Wnt11 (dilution, 1:500), GAPDH (dilution, 1:10,000), $\beta$-catenin (dilution, 1:200), GSK3 $\beta$ (dilution, 1:2,000), P-GSK3 $\beta$ (dilution, 1:800), $\beta$-actin (dilution, 1:200). The following day, the membrane was incubated with HRP-conjugated goat anti-rabbit (cat. no. 074-1506, KPL, Inc., Gaithersburg, MD, USA; dilution, 1:10,000) as secondary antibodies for $2 \mathrm{~h}$ at room temperature. The ChemiDoc Touch Imaging System (Bio-Rad Laboratories, Inc., Hercules, CA, USA) was used to detect the protein signals and analysis was conducted using Bandscan 4.3; BioMarin Pharmaceutical, Inc., San Rafearl, CA, USA).

Statistical analysis. Data are expressed as the mean \pm standard deviation. Differences between the two groups were analyzed using an unpaired Student's t-test. Linear correlation was applied to analyze the relationship between levels of Snail1
Table I. Clinical characteristics of patients in the SR and AF groups.

\begin{tabular}{lcc}
\hline Characteristics & $\mathrm{SR}(\mathrm{n}=9)$ & $\mathrm{AF}(\mathrm{n}=10)$ \\
\hline Sex ratio (Male/female) & $5 / 4$ & $5 / 5$ \\
Age (years) & $48.44 \pm 7.38$ & $48.40 \pm 7.96$ \\
LVEF (\%) & $54.56 \pm 6.65$ & $52.70 \pm 3.56$ \\
NYHA (II/III) & $4 / 5$ & $4 / 6$ \\
Smoker (yes/no) & $2 / 7$ & $2 / 8$ \\
LAD (mm) & $44.441 \pm 7.13$ & $54.50 \pm 5.02^{\mathrm{a}}$
\end{tabular}

Age, LVEF and LAD are presented as the mean \pm standard deviation of the mean. Sex and classification of heart function were compared using Fisher's exact test. ${ }^{\mathrm{a}} \mathrm{P}<0.05$ vs. SR group. SR, sinus rhythm; AF, atrial fibrillation; LVEF, Left Ventricular Ejection Fraction; NYHA, New York heart function classification; LAD, Left atrial diameter.

mRNA and CVF by Pearson's correlation coefficient. The value of $r$ represents the correlation between two variables; $r>0.5$ indicates a positive correlation. Differences between the sex and heart function of two groups were analyzed using Fisher's exact test. Differences between the age, LVEF and LAD of two groups were analyzed using an unpaired Student's t-test. SPSS 19.0 statistical software (IBM Corp., Armonk, NY, USA) was used to perform statistical analysis. $\mathrm{P}<0.05$ was considered to indicate a statistically significant difference.

\section{Results}

Patients with AF and RHD exhibit an expanded LAD. All patients participating in the present study received preoperative routine testing, including a blood test, chest X-ray, electrocardiogram and echocardiography. General information about patients was collected and it was determined that there were no significant differences in sex, age, left ventricular ejection fraction and New York heart function classification between the SR and AF groups (Table I). However, compared with the SR group, the patients with AF exhibited significantly expanded LAD $(\mathrm{P}<0.05$; Table I). This indicates that patients with AF and RHD have a larger LAD than patients with SR.

Patients with AF and RHD exhibit expansive atrial myocytes and accumulated collagen. To investigate the effect of AF on atrial structural remodeling, $H \& E$ and Masson staining were used to evaluate the extent of myocardial fibrosis (Fig. 1). $\mathrm{H} \& \mathrm{E}$ staining indicated that the size of the myocardial cells in the AF group was greater than those in the SR group (Fig. 1A). Myocardial fibrosis was evaluated by measuring the total amount of collagen in the interstitial spaces of the myocardial tissue and determining the CVF. Compared with the SR group, the AF group exhibited a disorder in the arrangement of interstitial collagen fibers (Fig. 1B). Furthermore, the CVF was significantly higher in the AF group compared with the SR group $(\mathrm{P}<0.05$; Fig. $1 \mathrm{C})$. These results indicate that patients with AF and RHD have expansive atrial myocytes and exhibit extensive collagen deposition in the atrial myocardium. 
A



Figure 1. Expansive atrial myocytes and accumulated collagen in the patients with AF and rheumatic heart disease. (A) Hematoxylin and eosin staining of the right atrial tissue of patients in the SR and AF groups. (B) Masson staining of the right atrial tissue of patients in the SR and AF groups. Magnification, $\mathrm{x} 400$. (C) Collagen volume fraction of the SR and AF groups. "P<0.05 vs. SR group. SR, sinus rhythm; AF, atrial fibrillation.

Elevated Snaill expression in the atrial myocardium of patients with $A F$ and RHD. To determine the location and expression of Snail1, deposition of Snaill in the myocardial tissues was measured using immunohistochemistry. The expression of Snail1 mRNA and protein was measured using RT-qPCR and western blotting, respectively. Immunohistochemical staining was performed in tissue sections to determine the distribution of Snaill; the results indicated that Snaill was primarily distributed in the vascular endothelial and interstitial cells and was almost undetectable in myocardial cells in the AF group. (Fig. 2A). Additionally, levels of Snaill mRNA and protein in the atrial tissues of patients with $\mathrm{AF}$ were significantly higher than those of the SR group $(\mathrm{P}<0.05$; Fig. $2 \mathrm{~B}$ and $\mathrm{C})$. These data demonstrate that levels of Snaill are increased in the myocardium of patients with AF and indicate that Snaill may be involved in the development of atrial fibrosis.

Snaill is positively correlated with atrial fibrosis in patients with $A F$ and RHD. To determine the association between Snaill expression and atrial fibrosis, a correlation analysis was performed. A positive correlation was identified between levels of Snail1 mRNA and CVF ( $\mathrm{r}=0.717$; $\mathrm{P}<0.001$; Fig. 3). This confirms that there is a positive association between Snaill expression and atrial fibrosis in patients with AF and suggests that Snaill may be developed as a novel biomarker to evaluate myocardial fibrosis for patients with AF and RHD in the future.

The Wnt signaling pathway may participate in the process of increased Snaill expression and atrial fibrosis in patients with $A F$ and $R H D$. Wnt signaling serves an important role in organ fibrosis, which is involved in renal, lung and liver fibrosis $(17,18,21)$. To determine whether the activity of the Wnt signaling pathway increased Snaill expression and atrial fibrosis in patients with $\mathrm{AF}$, the expression of proteins involved in the Wnt signaling pathway was investigated (Fig. 4). It was determined by western blotting that, compared with the SR group, the expression of Wnt1, 3a and 8a, which are involved in the canonical Wnt signaling pathway, were significantly increased in the AF group ( $\mathrm{P}<0.05$; Fig. 4B). Furthermore, the levels of Wnt5a and Wnt11, which are involved in the noncanonical Wnt signaling pathway, were also significantly higher in the AF group compared with the SR group $(\mathrm{P}<0.05$; Fig. 4B). It was also observed that the phosphorylation levels of GSK3 $\beta$, as well as the levels of $\beta$-catenin, were significantly elevated in the AF group ( $\mathrm{P}<0.05 ;$ Fig. $4 \mathrm{C})$. These results indicate that the Wnt signaling pathway, which is associated with the development of EMT, may also contribute to increased Snaill expression and the development of atrial fibrosis.

\section{Discussion}

In the present study, it was determined that the size of myocardial cells was more expansive and the degree of myocardial fibrosis was significantly increased in patients with AF. Additionally, levels of Snaill mRNA and protein were increased in patients with AF and Snail1 was primarily deposited in vascular endothelial and interstitial cells; expression in Snaill was low in myocardial cells. Correlation analysis determined that levels of Snail 1 mRNA were positively correlated with the degree of atrial fibrosis in patients with AF and RHD. Finally, the expression of factors involved in the canonical (Wnt1, 3a and 8a) and noncanonical Wnt signaling pathways (Wnt5a and 11) were significantly increased in the AF group and the phosphorylation levels of GSK3 $3 \beta$ and $\beta$-catenin were also elevated in the AF group. These results indicate that increased Snaill expression is positively associated with the degree of atrial fibrosis. Furthermore, they suggest that the Wnt signaling pathway, which is associated with the development of EMT, may also contribute to increased Snaill expression and atrial fibrosis in patients with AF and RHD.

Atrial fibrosis is the primary process by which atrial structure remodeling occurs and serves a key role in the development and persistence of AF (3). Therefore, attenuating atrial fibrosis to inhibit atrial structure remodeling is crucial to prevent the onset and development of AF. Furthermore, atrial fibrosis is mediated by cardiac fibroblasts and epithelial cells, and endothelial cells can be transformed to fibroblasts during the EMT, which performs important roles in pulmonary fibrosis, renal fibrosis, cardiac hypertrophy and cardiac fibrosis (10-14). Therefore, inhibiting the EMT to attenuate cardiac fibrosis may be an important method of preventing the development of AF.

Snaill is a member of the Snail family, which is a specific marker of the EMT and serves an important role in tissue fibrosis, including kidney and liver fibrosis $(13,16,22-24)$. It was demonstrated that during liver fibrosis, Snaill expression is upregulated and that it serves a key role in the progression of liver fibrosis by upregulating the biosynthesis of the extracellular matrix and promoting chronic inflammatory 


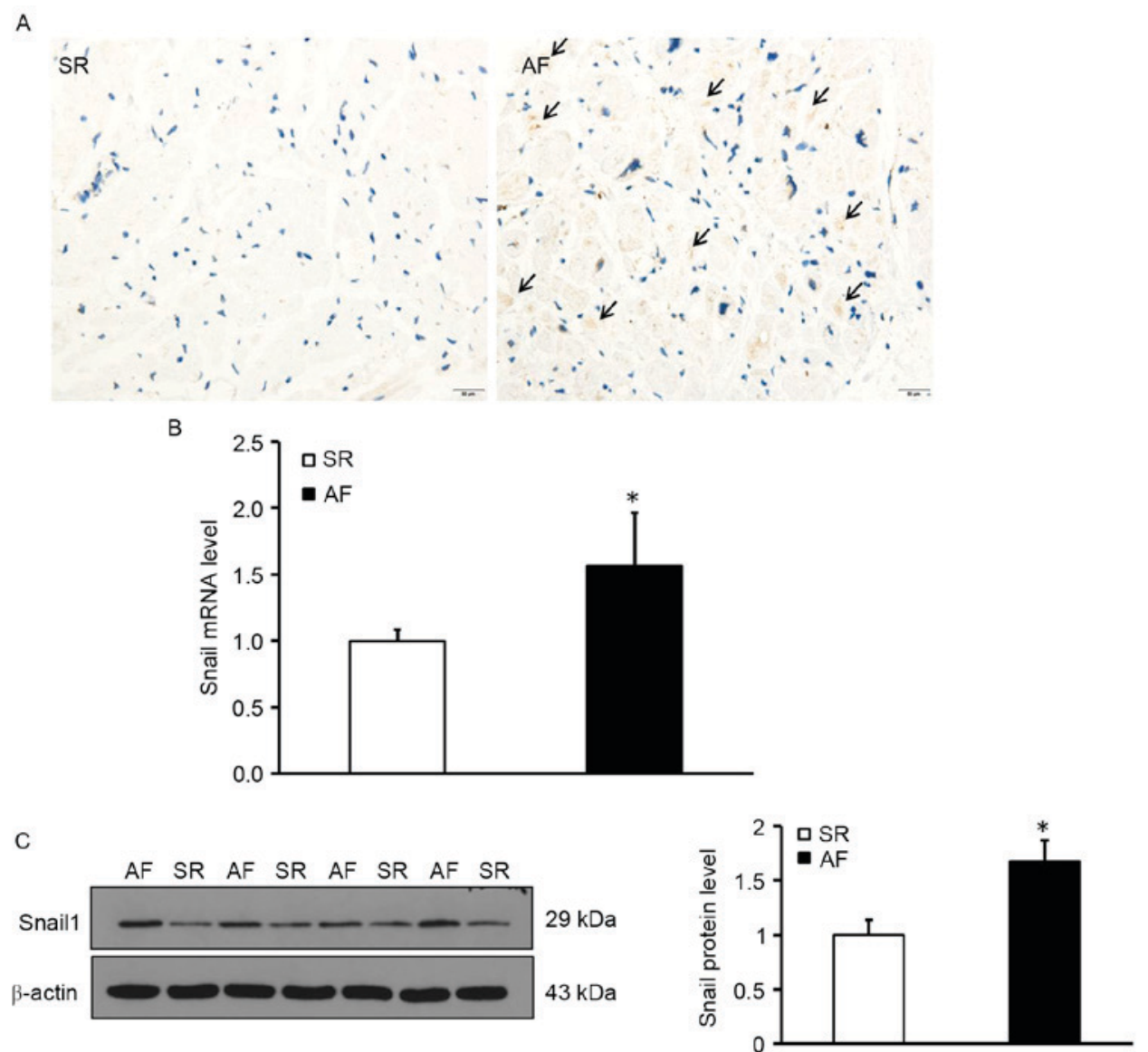

Figure 2. Elevated Snail1 levels in the atrial myocardium of patients with $\mathrm{AF}$ and rheumatic heart disease. (A) Representative immunohistochemistical staining images of Snaill in the SR and AF groups. Black arrows indicate positive expression of Snaill. Magnification, x400. (B) The expression of Snaill mRNA in the SR and AF groups. (C) The expression of Snaill protein in the SR and AF groups. Left, representative western blot presenting the levels of Snaill protein; Right, quantitative analysis of the levels of Snail1 protein in the SR and AF groups. "P<0.05 vs. SR group. SR, sinus rhythm; AF, atrial fibrillation; RHD, rheumatic heart disease.

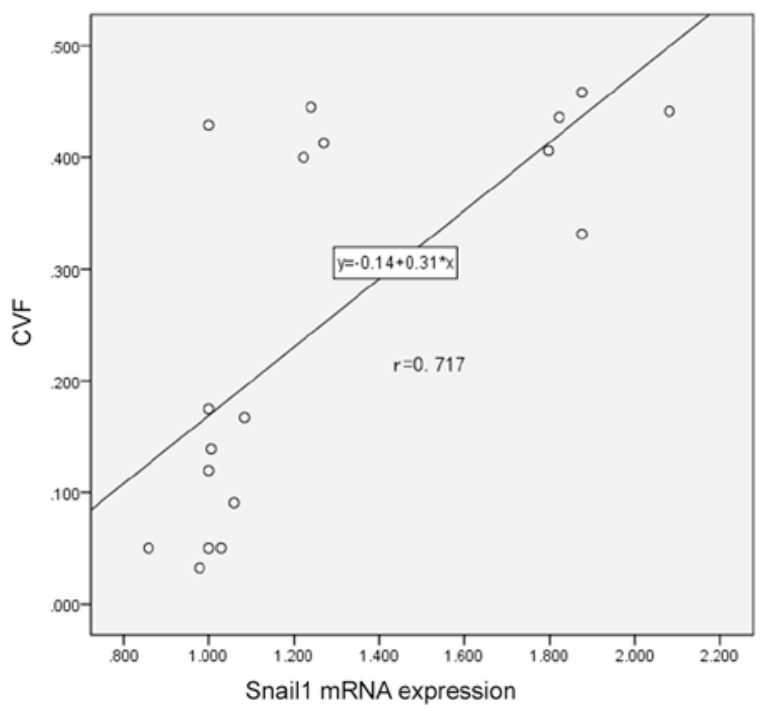

Figure 3. Correlation analysis of Snaill mRNA expression and atrial fibrosis. Snail1 is positively correlated with atrial fibrosis in patients with atrial fibrillation and rheumatic heart disease. CVF, collagen volume fraction.

responses (24). Additionally, it was determined that the pattern of Snaill expression is spatial and temporal and may increase following injuries that occur during fibrogenesis $(16,25)$. In the present study, it was demonstrated that Snaill expression was markedly increased in the myocardium and that Snaill was primarily deposited in the vascular endothelial and interstitial cells. Furthermore, it was determined that Snaill was positively correlated with the degree of atrial fibrosis in patients with AF and RHD. These results indicate that the EMT may serve an important role in the development of atrial fibrosis in patients with AF and RHD.

Additionally, Wnt signaling is one of the most important signaling pathways involved in tissue fibrosis and regulates the adherence and migration of cells (15). It has been demonstrated that $\mathrm{Wnt} / \beta$-catenin signaling serves an important role in renal and pulmonary fibrosis $(17,18)$. Furthermore, it has been determined that the EMT may be induced by Wnt signaling that is activated by cardiac injury (26). Therefore, the present study also measured changes in the levels of proteins involved in Wnt signaling. It was demonstrated that, compared with the SR group, levels of Wnt1, 3a and 8a involved in the canonical Wnt signaling pathway and levels of Wnt5a and Wnt11 involved in the noncanonical Wnt signaling pathway were significantly higher in the AF group compared with the SR group. Additionally, the phosphorylation level of GSK3 $\beta$ and level of $\beta$-catenin were significantly increased in the AF group. It was thus determined that the canonical and noncanonical 
A



B

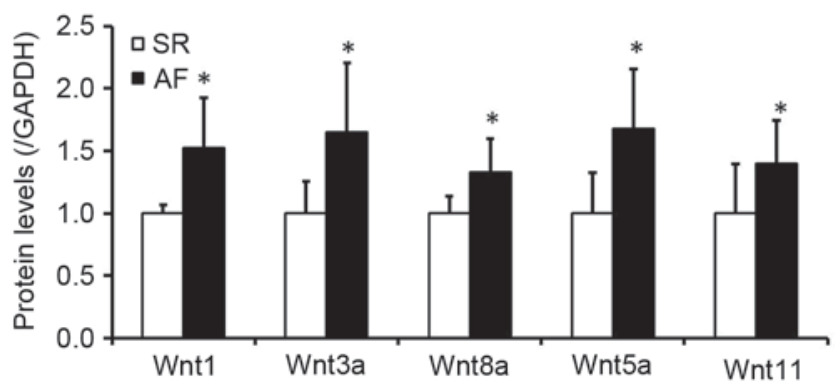

C


Figure 4. The Wnt signaling pathway may participate in the process of increased Snaill expression and atrial fibrosis in patients with AF and rheumatic heart disease. (A) Representative western blot presenting the levels of Wnt1, Wnt3a, Wnt5a, Wnt8a and Wnt11 protein. (B) Quantitative analysis of Wnt1, Wnt3a, Wnt5a, Wnt8a and Wnt11 expression in the SR and AF groups. (C) Left, representative western blot indicating the levels of GSK3 $\beta$, P-GSK3 $\beta$ and $\beta$-catenin expression. Right, quantitative analysis of levels of GSK3 $\beta$, P-GSK3 $\beta$ and $\beta$-catenin in the SR and AF groups. "P $<0.05$ vs. SR group. SR, sinus rhythm; AF, atrial fibrillation; GSK3 $\beta$, glycogen synthase kinase $3 \beta$; P-GSK3 $\beta$, phosphorylated glycogen synthase kinase $3 \beta$.

Wnt signaling pathways were activated in the myocardium of patients with AF. Previous studies have demonstrated that the Snaill-induced EMT occurs, at least in part, due to a decrease in E-cadherin transcription that stimulates the development of epithelial phenotypic cells with adhesive and polarity properties. These cells then gradually transform into loose and activated mesenchymal cells and stimulate the development of tissue fibrosis $(22,27)$. These results indicate that the Wnt signaling pathway is associated with the development of EMT and may participate in the process of increased Snaill and atrial fibrosis in patients with AF and RHD.

In conclusion, the present study demonstrated that Snaill may be involved in the development and maintenance of atrial fibrosis in patients with AF and RHD, and that Snaill may be used as a novel biomarker to evaluate atrial fibrosis in patients with AF and RHD in the future. Furthermore, the Wnt signaling pathway associated with the development of EMT may increase Snaill expression and atrial fibrosis in patients with AF and RHD. Novel drugs that inhibit the expression and/or function of Snail1, or block the Wnt signaling pathway may prevent atrial fibrosis in patients with AF and RHD. However the sample size of the current study was relatively small and further studies with a larger sample size or involving multiple centers are required to further elucidate this mechanism of action. Future in vitro or in vivo studies are also required to determine how the Wnt signaling pathway regulates Snail1 expression and induces the development of EMT.

\section{Acknowledgements}

The authors wish to thank Professors Zhiwei Wang and Jun Xia, who helped out with the sample collection and all doctors in the Department of Cardiology and the Cardiovascular Research Institute of Renmin Hospital of Wuhan University for their expert technical assistance and advice. The present study was supported by grants from the National Natural Science Foundation of China (no. 81170085) and the Fundamental Research Funds for the Central Universities (no. 2042016kf0074). 


\section{References}

1. Li M, Yi X, Ma L and Zhou Y: Hepatocyte growth factor and basic fibroblast growth factor regulate atrial fibrosis in patients with atrial fibrillation and rheumatic heart disease via the mitogen-activated protein kinase signaling pathway. Exp Ther Med 6: 1121-1126, 2013.

2. Jalife $\mathrm{J}$ and Kaur K: Atrial remodeling, fibrosis, and atrial fibrillation. Trends Cardiovasc Med 25: 475-484, 2015.

3. Tan AY and Zimetbaum P: Atrial fibrillation and atrial fibrosis. J Cardiovasc Pharmacol 57: 625-629, 2011.

4. Sun Y, Huang ZY, Wang ZH, Li CP, Meng XL, Zhang YJ, Su F and Ma N: TGF- $\beta 1$ and TIMP-4 regulate atrial fibrosis in atrial fibrillation secondary to rheumatic heart disease. Mol Cell Biochem 406: 131-138, 2015.

5. Zhang YJ, Ma N, Su F, Liu H and Mei J: Increased TRPM6 expression in atrial fibrillation patients contribute to atrial fibrosis. Exp Mol Pathol 98: 486-490, 2015.

6. Miyasato SK, Loeffler J, Shohet R, Zhang J, Lindsey M and Le Saux CJ: Caveolin-1 modulates TGF- $\beta 1$ signaling in cardiac remodeling. Matrix Biol 30: 318-329, 2011.

7. Zhang L, Huang B, Scherlag BJ, Ritchey JW, Embi AA, Hu J, Hou Y and Po SS: Structural changes in the progression of atrial fibrillation: Potential role of glycogen and fibrosis as perpetuating factors. Int J Clin Exp Pathol 8: 1712-1718, 2015.

8. Kiryu M, Niwano S, Niwano H, Kishihara J, Aoyama Y, Fukaya H, Masaki Y and Izumi T: Angiotensin II-mediated up-regulation of connective tissue growth factor promotes atrial tissue fibrosis in the canine atrial fibrillation model. Europace 14 1206-1214, 2012

9. Wang R, Yi X, Li X and Jiang X: Fibroblast growth factor-21 is positively associated with atrial fibrosis in atrial fibrillation patients with rheumatic heart disease. Int J Clin Exp Pathol 8 : 14901-14908, 2015.

10. Lamouille S, Xu J and Derynck R: Molecular mechanisms of epithelial-mesenchymal transition. Nat Rev Mol Cell Biol 15: 178-196, 2014

11. Baum B, Settleman J and Quinlan MP: Transitions between epithelial and mesenchymal states in development and disease. Semin Cell Dev Biol 19: 294-308, 2008.

12. Hashimoto $\mathrm{N}$, Phan SH, Imaizumi K, Matsuo $\mathrm{M}$, Nakashima H, Kawabe T, Shimokata K and Hasegawa Y: Endothelial-mesenchymal transition in bleomycin-induced pulmonary fibrosis. Am J Respir Cell Mol Biol 43: 161-172, 2010

13. Tennakoon $A H$, Izawa $T$, Kuwamura $M$ and Yamate $J$ : Pathogenesis of Type 2 Epithelial to Mesenchymal Transition (EMT) in renal and hepatic fibrosis. J Clin Med 5: pii:E4, 2015.

14. Widyantoro B, Emoto N, Nakayama K, Anggrahini DW, Adiarto S, Iwasa N, Yagi K, Miyagawa K, Rikitake Y, Suzuki T, et al: Endothelial cell-derived endothelin-1 promotes cardiac fibrosis in diabetic hearts through stimulation of endothelial-to-mesenchymal transition. Circulation 121: 2407-2418, 2010 .
15. Barrallo-Gimeno A and Nieto MA: The Snail genes as inducers of cell movement and survival: Implications in development and cancer. Development 132: 3151-3161, 2005.

16. Liu Y, Du J, Zhang J, Weng M, Li X, Pu D, Gao L, Deng S, $\mathrm{Xia} S$ and She Q: Snaill is involved in de novo cardiac fibrosis after myocardial infarction in mice. Acta Biochim Biophys Sin (Shanghai) 44: 902-910, 2012.

17. Dang Y, Liu B, Xu P, Zhu P, Zhai Y, Liu M and Ye X: Gpr48 deficiency induces polycystic kidney lesions and renal fibrosis in mice by activating Wnt signal pathway. PLoS One 9: e89835, 2014.

18. Kim TH, Kim SH, Seo JY, Chung H, Kwak HJ, Lee SK, Yoon HJ, Shin DH, Park SS and Sohn JW: Blockade of the Wnt/ $\beta$-catenin pathway attenuates bleomycin-induced pulmonary fibrosis. Tohoku J Exp Med 223: 45-54, 2011.

19. Ge WS, Wang YJ, Wu JX, Fan JG, Chen YW and Zhu L: $\beta$-catenin is overexpressed in hepatic fibrosis and blockage of Wnt/ $\beta$-catenin signaling inhibits hepatic stellate cell activation. Mol Med Rep 9: 2145-2151, 2014.

20. Livak KJ and Schmittgen TD: Analysis of relative gene expression data using real-time quantitative PCR and the 2(-Delta Delta C(T)) method. Methods 25: 402-408, 2001.

21. Yu F, Lu Z, Huang K, Wang X, Xu Z, Chen B, Dong P and Zheng J: MicroRNA-17-5p-activated Wnt/ $\beta$-catenin pathway contributes to the progression of liver fibrosis. Oncotarget 7 : 81-93, 2016.

22. Boutet A, De Frutos CA, Maxwell PH, Mayol MJ, Romero J and Nieto MA: Snail activation disrupts tissue homeostasis and induces fibrosis in the adult kidney. EMBO J 25: 5603-5613, 2006.

23. Xu-Dubois YC, Galichon P, Brocheriou I, Baugey E, Morichon R, Jouanneau C, Ouali N, Rondeau E and Hertig A: Expression of the transcriptional regulator snaill in kidney transplants displaying epithelial-to-mesenchymal transition features. Nephrol Dial Transplant 29: 2136-2144, 2014.

24. Rowe RG, Lin Y, Shimizu-Hirota R, Hanada S, Neilson EG, Greenson JK and Weiss SJ: Hepatocyte-derived Snaill propagates liver fibrosis progression. Mol Cell Biol 31: 2392-2403, 2011.

25. Zhou B, Honor LB, He H, Ma Q, Oh JH, Butterfield C, Lin RZ, Melero-Martin JM, Dolmatova E, Duffy HS, et al: Adult mouse epicardium modulates myocardial injury by secreting paracrine factors. J Clin Invest 121: 1894-1904, 2011.

26. Duan J, Gherghe C, Liu D, Hamlett E, Srikantha L, Rodgers L, Regan JN, Rojas M, Willis M, Leask A, et al: Wnt1/ $\beta$ catenin injury response activates the epicardium and cardiac fibroblasts to promote cardiac repair. EMBO J 31:429-442, 2012.

27. Ohnuki K, Umezono T, Abe M, Kobayashi T, Kato M, Miyauchi M, Yamamoto N, Kimura M, Toyoda M and Suzuki D: Expression of transcription factor Snail and tubulointerstitial fibrosis in progressive nephropathy. J Nephrol 25: 233-239, 2012. 